\title{
Transverse effects in the laser threshold due to electronic-vibrational coupling
}

\author{
Oscar G. Calderón* and Isabel Gonzalo \\ Departamento de Optica, Facultad de Ciencias Físicas, Universidad Complutense de Madrid, Ciudad Universitaria s/n, \\ 28040 Madrid, Spain
}

(Received 20 February 1997; revised manuscript received 3 October 1997)

\begin{abstract}
Transverse effects in the laser threshold, originated by electronic-vibrational coupling in the active centers, are analyzed theoretically by means of the semiclassical two-level Maxwell-Bloch equations. A single longitudinal mode is considered. It is found that the first laser threshold suffers modifications depending on the electronic-vibrational coupling strength. This coupling imposes certain conditions for the selection of a particular transverse spatial state and provides the minimum wavelength that can appear in the transverse pattern. The nature of the bifurcation and the stability of the homogeneous and critical traveling waves are analyzed. [S1050-2947(98)08502-3]

PACS number(s): 42.60.Mi, 47.20.Ky, 05.45.+b
\end{abstract}

\section{INTRODUCTION}

In the electronic excitation of molecules or crystals by radiation, vibrational couplings may modify significantly the behavior of the system. Phonon or vibrational effects have been taken into account in nonlinear optics [1-5]. In particular, phonon effects on optical bistability were shown to lead to a new type of optical bistability [4-7]. Effects of the electronic-vibrational coupling in a single mode laser without transverse effects have been recently studied [8]. It was found that the region of laser oscillation shifts to a lower frequency and suffers modifications so that for particular conditions there are pump values above which laser emission can disappear.

One of the most interesting topics at the present is the transverse effects in lasers [9-14]. The single-longitudinalmode laser has been a useful laboratory to study transverse phenomena without the influence of other degrees of freedom [15].

Concerning the transverse effects at threshold, an analysis of the laser bifurcation [16-19] shows that for negative detuning, the mode with the biggest growth rate at threshold is homogeneous $(k=0)$, whereas for positive detuning, a traveling wave is expected above threshold. Its corresponding wave vector is related to the detuning $\Delta$ and to the diffraction coefficient $v$ by $k=\sqrt{\Delta / v}$ [17].

In this paper we study theoretically the modifications of transverse effects in the laser threshold originated by electronic-vibrational coupling in the active centers. A single longitudinal mode is considered. The description is made by means of the semiclassical two-level Maxwell-Bloch equations, assuming the rotating wave and the slowly varying amplitude approximations. The electronic-vibrational coupling considered is more general than the coupling assumed in the previous work [8]. The neutral stability curve obtained in the present work, in the linear stability analysis of the nonlasing state, presents some features that are similar to those obtained in the case of a Raman laser model [18]. In our case the curve depends on the electronic-vibrational cou-

*Electronic address: oscargc@eucmax.sim.ucm.es pling strengths. We analyze how this dependence imposes certain conditions for the selection of a particular spatial state at the first laser threshold. We found that the vibrational coupling considered provides the minimum spatial scale (wavelength) that can appear in the transverse pattern. It complements the result of a previous work [20] where the spatial diffusion term found provides also a cutoff value for the size of the spatial structures that can appear in the system.

The nature of the bifurcation at the first laser threshold is analyzed. In the case without electronic-vibrational coupling, the bifurcation is always supercritical [18], but in our case, we find that it may be supercritical or subcritical, depending on the laser parameters.

We analyze the stability of the two patterns selected at the first laser threshold (the critical traveling wave and the homogeneous wave), the homogeneous solution being the only one that can survive for high pumping values.

\section{THE MODEL}

Our system consists of $N$ identical molecules per unit volume inserted in a cavity. The length of the cavity is tuned to a single longitudinal mode of frequency $\omega$. Each molecule is modeled by two electronic levels only, the ground state $|1\rangle$ and the excited state $|2\rangle$ with respective energies $E_{1}$ and $E_{2}$ in the optical range, connected by one photon transition so that $\hbar \omega \approx E_{2}-E_{1}$.

We shall consider that the active electrons of the molecules interact with the vibrational normal modes of the nuclei (phonons if the molecule is a big polymer or we deal with a crystal) by means of a simplified model.

The Hamiltonian of the electronic system is

$$
H=H^{0}+H^{e-r}+H^{e-v} .
$$

$H^{0}$ is the free electronic Hamiltonian whose eigenstates are $|1\rangle$ and $|2\rangle$. The electronic wave function is then

$$
|\psi(t)\rangle=C_{1}(t) e^{-i E_{1} t / \hbar}|1\rangle+C_{2}(t) e^{-i E_{2} t / \hbar}|2\rangle .
$$

The electric dipole interaction with the radiation is given by $H^{e-r}=-\mu E$, with $\mu \equiv-e r$, $e$ being the absolute value of 
the charge of the electron, assuming that the radiation is linearly polarized, with the electric field $\vec{E}$ in the same direction as the transition and permanent dipoles. $H^{e-v}$ is the electron-vibration Hamiltonian, where we assume a linear coupling between the electronic states and the vibrational normal modes. To analyze better this term it is first written, following Refs. [21,22,6,7], as

$$
\begin{aligned}
H^{e-v}= & \sum_{s_{1}} \hbar \omega_{s_{1}}\left(u_{s_{1}}^{*} b_{s_{1}}+u_{s_{1}} b_{s_{1}}^{\dagger}\right)|1\rangle\langle 1|+\sum_{s_{2}} \hbar \omega_{s_{2}}\left(u_{s_{2}}^{*} b_{s_{2}}\right. \\
& \left.+u_{s_{2}} b_{s_{2}}^{\dagger}\right)|2\rangle\langle 2|
\end{aligned}
$$

where $b_{s_{1}}, b_{s_{1}}^{\dagger}$, and $b_{s_{2}}, b_{s_{2}}^{\dagger}$ are the vibrational quanta annihilation and creation operators for the ground and excited states, respectively, $\omega_{s_{1}}$ and $\omega_{s_{2}}$ are the vibrational frequencies, $\omega_{s_{1}}, \omega_{s_{2}} \ll \omega$, and $u_{s_{1}}$ is dependent on the interaction strength and related to the relative displacement of the equilibrium positions of the nuclei after the molecule reaches the ground level $|1\rangle$ coming from level $|2\rangle$, and in an analogous way, $u_{s_{2}}$ is related to the relative displacement of the equilibrium positions of the nuclei after the molecule reaches the excited level $|2\rangle$ coming from level $|1\rangle$.
As the lifetime of the electronic states is much longer than the evolution time of the nuclei to the new equilibrium positions, we can evaluate the quantum mechanical average of $H^{e-v}$ factoring the electronic and nuclei variables, that is, $\left|C_{2}\right|^{2}$ and $\left|C_{1}\right|^{2}$ can be considered to be constant while the nuclei evolve to the new equilibrium positions:

$$
\begin{aligned}
\left\langle H^{e-v}\right\rangle_{\psi \otimes v}= & \sum_{s_{1}} \hbar \omega_{s_{1}}\left|C_{1}\right|^{2}\left\langle u_{s_{1}}^{*} b_{s_{1}}+u_{s_{1}} b_{s_{1}}^{\dagger}\right\rangle_{v} \\
& +\sum_{s_{2}} \hbar \omega_{s_{2}}\left|C_{2}\right|^{2}\left\langle u_{s_{2}}^{*} b_{s_{2}}+u_{s_{2}} b_{s_{2}}^{\dagger}\right\rangle_{v} .
\end{aligned}
$$

Taking the new equilibrium coordinates as the new origin of reference, the quantum vibrational operator for the mode reads

$$
\bar{b}_{s_{1}}=b_{s_{1}}+\left|C_{1}\right|^{2} u_{s_{1}}, \quad \bar{b}_{s_{2}}=b_{s_{2}}+\left|C_{2}\right|^{2} u_{s_{2}},
$$

whereupon the averaged electron-vibration interaction can be written as

$$
\begin{aligned}
\left\langle H^{e-v}\right\rangle_{\psi \otimes v} & =\sum_{s_{1}} \hbar \omega_{s_{1}}\left|C_{1}\right|^{2}\left\langle\left(u_{s_{1}}^{*} \bar{b}_{s_{1}}+u_{s_{1}} \bar{b}_{s_{1}}^{\dagger}\right)-2\left|C_{1}\right|^{2}\left|u_{s_{1}}\right|^{2}\right\rangle_{v}+\sum_{s_{2}} \hbar \omega_{s_{2}}\left|C_{2}\right|^{2}\left\langle\left(u_{s_{2}}^{*} \bar{b}_{s_{2}}+u_{s_{2}} \overline{b_{s_{2}}^{\dagger}}\right)-2\left|C_{2}\right|^{2}\left|u_{s_{2}}\right|^{2}\right\rangle_{v} \\
& =\sum_{s_{1}}-2 \hbar \omega_{s_{1}}\left|C_{1}\right|^{4}\left|u_{s_{1}}\right|^{2}+\sum_{s_{2}}-2 \hbar \omega_{s_{2}}\left|C_{2}\right|^{4}\left|u_{s_{2}}\right|^{2}=-(1-d) \hbar f_{1}\left|C_{1}\right|^{2}-(1+d) \hbar f_{2}\left|C_{2}\right|^{2},
\end{aligned}
$$

where $\quad f_{1} \equiv \Sigma_{s_{1}} f_{s_{1}}, \quad f_{2} \equiv \Sigma_{s_{2}} f_{s_{2}}, \quad f_{s_{1}} \equiv \omega_{s_{1}}\left|u_{s_{1}}\right|^{2}, \quad f_{s_{2}}$ $\equiv \omega_{s_{2}}\left|u_{s_{2}}\right|^{2}$ have frequency dimension and $1+d=2\left|C_{2}\right|^{2}$, $1-d=2\left|C_{1}\right|^{2}$, with $d \equiv\left|C_{2}\right|^{2}-\left|C_{1}\right|^{2}$ being the population inversion per molecule. The effective electron-vibration Hamiltonian acting only on the electronic state $|\psi\rangle$ is then

$$
H_{\text {eff }}^{e-v}=-(1-d) \hbar f_{1}|1\rangle\left\langle 1\left|-(1+d) \hbar f_{2}\right| 2\right\rangle\langle 2| .
$$

It must be noted that the values of the frequencies $\omega_{s_{1}}$, $\omega_{s_{2}}$ are between $\sim 10^{11} \mathrm{~s}^{-1}$ (some bending modes in large plane molecules) and $\sim 10^{13} \mathrm{~s}^{-1}$. As the dimensionless parameters $\left|u_{s_{1}}\right|^{2},\left|u_{s_{2}}\right|^{2}$ may take values from 1 to 20 approximately [23], the values of $f_{1}, f_{2}$ can be of the order of $10^{12}$ or $10^{13} \mathrm{~s}^{-1}$.

To obtain the evolution equations of the system we follow a well-known procedure [24]. From the Schrödinger equation for the function given by Eq. (2), the time evolution equations for $C_{1}$ and $C_{2}$ are

$$
\begin{gathered}
i \hbar \frac{d C_{1}}{d t}=E \mu_{11} C_{1}+E \mu_{12} C_{2} e^{-i \omega_{12} t}-(1-d) \hbar f_{1} C_{1}, \\
i \hbar \frac{d C_{2}}{d t}=E \mu_{21} C_{1} e^{i \omega_{12} t}+E \mu_{22} C_{2}-(1+d) \hbar f_{2} C_{2},
\end{gathered}
$$

where $\mu_{i j} \equiv\langle i|e r| j\rangle$ and $\omega_{12} \equiv\left(E_{2}-E_{1}\right) / \hbar$. The microscopic polarization is

$$
p=\langle\psi|-e r| \psi\rangle=\left(p^{+}+p^{-}\right)+\mu_{11}\left|C_{1}\right|^{2}+\mu_{22}\left|C_{2}\right|^{2},
$$

where $p^{+} \equiv-\mu_{12} C_{1}^{*} C_{2} e^{-i \omega_{12} t}$ and $p^{-}=p^{+*}$. Taking the time derivative of $p^{+}$and of the population inversion per molecule, $d$, using the expressions (8), (9), multiplying by $N$ to introduce the macroscopic variables $P=N p, D=N d$, and including phenomenologically the relaxation terms for the polarization and population inversion, we obtain

$$
\begin{aligned}
\frac{\partial P^{+}}{\partial t}=- & \left(\gamma_{\perp}+i \omega_{12}\right) P^{+}-i \frac{E\left(\mu_{22}-\mu_{11}\right)}{\hbar} P^{+}+i\left(f_{2}-f_{1}\right. \\
+ & \left.\frac{D}{N}\left(f_{2}+f_{1}\right)\right) P^{+}-\frac{i\left|\mu_{12}\right|^{2} E D}{\hbar} \\
& \frac{\partial D}{\partial t}=\gamma_{\|}\left(D_{0}-D\right)-\frac{2 i E}{\hbar}\left(P^{+}-P^{-}\right)
\end{aligned}
$$

where $D_{0}$ is the population inversion induced by the pumping, $\gamma_{\perp}^{-1}$ is the depolarization time of the dipoles induced by 
the radiation, and $\gamma_{\|}^{-1}$ is the relaxation time of the population inversion. The electric radiation field into the cavity is given by the Maxwell wave equation

$$
\Delta E-\mu_{0} \sigma_{c} \frac{\partial E}{\partial t}-\frac{1}{c^{2}} \frac{\partial^{2} E}{\partial t^{2}}=\mu_{0} \frac{\partial^{2} P}{\partial t^{2}} \quad \text { (I.S. units), }
$$

where $\sigma_{c}$ stands for the effective cavity losses and $\mu_{0}$ is the vacuum magnetic permeability. The field is assumed to be of the form $E=E_{0}^{+}(x, y, t) \exp (i k z-i \omega t)+$ c.c. Establishing the one-photon resonance condition $\left(\omega \approx \omega_{12}\right)$, using the rotating wave, and the slowly varying amplitude approximations, Eqs. (11)-(13) transform into

$$
-i \frac{c^{2}}{\omega} \Delta_{\perp} E_{0}^{+}+2 \frac{\partial E_{0}^{+}}{\partial t}=-\mu_{0} c^{2} \sigma_{c} E_{0}^{+}+i \mu_{0} c^{2} \omega P_{0}^{+},
$$

$$
\begin{gathered}
\frac{\partial P_{0}^{+}}{\partial t}=-\left(\gamma_{\perp}+i \omega_{12}-i \omega\right) P_{0}^{+}+i P_{0}^{+}\left(f_{2}-f_{1}+\frac{D}{N}\left(f_{2}+f_{1}\right)\right) \\
-\frac{i\left|\mu_{12}\right|^{2} E_{0}^{+} D}{\hbar} \\
\frac{\partial D}{\partial t}=\gamma_{\|}\left(D_{0}-D\right)-\frac{2 i}{\hbar}\left(E_{0}^{-} P_{0}^{+}-E_{0}^{+} P_{0}^{-}\right)
\end{gathered}
$$

where $P_{0}^{+}$is the polarization amplitude and $\Delta_{\perp} \equiv \partial^{2} / \partial x^{2}$ $+\partial^{2} / \partial y^{2}$ the transversal Laplacian. To obtain dimensionless and simplified equations, we define $\tau \equiv \gamma_{\perp} t, \vec{\rho}=(\xi, \eta)$ $\equiv(x, y) / b$ ( $b$ being the transverse size of the resonator), $\alpha$ $\equiv N \omega\left|\mu_{12}\right|^{2} /\left(2 \epsilon_{0} c \hbar \gamma_{\perp}\right)$ and the following variable changes:

$$
\begin{gathered}
P_{0}^{+}=-\frac{i N\left|\mu_{12}\right| \sigma_{c}}{4 \epsilon_{0} c \alpha} \sqrt{\frac{\gamma_{\|}}{\gamma_{\perp}}} P_{s}, \quad E_{0}^{+}=\frac{\hbar \sqrt{\gamma_{\|} \gamma_{\perp}}}{2\left|\mu_{12}\right|} E_{s}, \\
D=\frac{N \sigma_{c}}{2 \epsilon_{0} c \alpha} D_{s}, \quad D_{0}=\frac{N \sigma_{c}}{2 \epsilon_{0} c \alpha} r,
\end{gathered}
$$

where $P_{s}, E_{s}, D_{s}$, and $r$, are the new dimensionless magnitudes. In terms of these new variables Eqs. (14)-(16) take finally the form

$$
\begin{gathered}
-i v \Delta_{\perp} E_{s}+\frac{\partial E_{s}}{\partial \tau}=\sigma\left(P_{s}-E_{s}\right), \\
\frac{\partial P_{s}}{\partial \tau}=-(1+i \delta) P_{s}+i a \sigma D_{s} P_{s}+D_{s} E_{s}, \\
\frac{\partial D_{s}}{\partial \tau}=-\gamma\left[D_{s}-r+\frac{1}{2}\left(E_{s}^{*} P_{s}+E_{s} P_{s}^{*}\right)\right],
\end{gathered}
$$

where $\quad v \equiv c \lambda /\left(4 \pi b^{2} \gamma_{\perp}\right) \quad$ (with $\left.\quad \lambda=2 \pi c / \omega\right), \quad \sigma$ $\equiv \sigma_{c} /\left(2 \epsilon_{0} \gamma_{\perp}\right), \quad \delta \equiv\left(\omega_{12}-\omega-f_{2}+f_{1}\right) / \gamma_{\perp} \equiv \Delta-\left(f_{2}\right.$ $\left.-f_{1}\right) / \gamma_{\perp} \quad\left[\right.$ with $\left.\Delta \equiv\left(\omega_{12}-\omega\right) / \gamma_{\perp}\right], a \equiv\left(f_{2}+f_{1}\right) /(c \alpha), \gamma$ $\equiv \gamma_{\|} / \gamma_{\perp}$, and the normalized pumping $r$ $\equiv 2 \epsilon_{0} c \alpha D_{0} /\left(N \sigma_{c}\right)$. This system of equations is similar to that of Maxwell-Bloch equations, except for the nonlinear term $i a \sigma D_{s} P_{s}$ and the term $i\left(\left(f_{2}-f_{1}\right) / \gamma_{\perp}\right) P_{s}$, both in the polarization equation. The factor $\left(f_{2}-f_{1}\right) / \gamma_{\perp}$ acts as a de- tuning originated by the vibrational coupling strengths $f_{1}$ and $f_{2}$. The factor $a \sigma D_{s}$ depends on the population inversion and acts as a dynamic detuning.

\section{LINEAR STABILITY ANALYSIS OF THE NONLASING SOLUTION}

Let us analyze the stability of the nonlasing solution $E_{s}$ $=0, P_{s}=0, D_{s}=r$. By linearizing about this trivial solution and expanding the variables as a Fourier series of transversal modes of wave vectors $k$, we get for each set of Fourier components $\left(\delta E_{k}, \delta P_{k}, \delta D_{k}\right)$ the following system:

$$
\begin{gathered}
\frac{\partial\left(\delta E_{k}\right)}{\partial \tau}=-i v k^{2}\left(\delta E_{k}\right)+\sigma\left(\left(\delta P_{k}\right)-\left(\delta_{k} E\right)\right) \\
\frac{\partial\left(\delta P_{k}\right)}{\partial \tau}=-(1+i \delta)\left(\delta P_{k}\right)+i a \sigma r\left(\delta P_{k}\right)+r\left(\delta E_{k}\right) \\
\frac{\partial\left(\delta D_{k}\right)}{\partial \tau}=-\gamma\left(\delta D_{k}\right)
\end{gathered}
$$

which yields the three eigenvalues

$$
\begin{gathered}
\lambda_{3}=-\gamma, \\
\lambda_{ \pm}=-\frac{1+\sigma+i\left(\delta-a \sigma r+v k^{2}\right)}{2} \\
\quad \pm \sqrt{\left(\frac{1-\sigma+i\left(\delta-a \sigma r-v k^{2}\right)}{2}\right)^{2}+\sigma r .}
\end{gathered}
$$

The trivial solution is linearly unstable if one of the eigenvalues has a positive real part. The expression under the square root is a complex number, which will be denoted by $(\chi+i \xi)^{2}$, with $\chi \geqslant 0$, namely,

$$
(\chi+i \xi)^{2} \equiv\left(\frac{1-\sigma+i\left(\delta-a \sigma r-v k^{2}\right)}{2}\right)^{2}+\sigma r .
$$

It can be seen that the trivial solution is unstable if and only if the real part of Eq. (26) is positive, i.e., $-[(1+\sigma) / 2]$ $\pm \chi>0$, equivalent to $\chi^{2}>[(1+\sigma) / 2]^{2}$. Equating the real and imaginary parts of both sides of Eq. (27) we find that $\chi$ satisfies

$$
\begin{aligned}
F\left(\chi^{2}\right) \equiv & \chi^{4}-\left[\left(\frac{1-\sigma}{2}\right)^{2}-\left(\frac{\delta-a \sigma r-v k^{2}}{2}\right)^{2}+\sigma r\right] \chi^{2} \\
& -\left(\frac{1-\sigma}{2}\right)^{2}\left(\frac{\delta-a \sigma r-v k^{2}}{2}\right)^{2}=0
\end{aligned}
$$

This expression can be interpreted as a function of a variable $F\left(\theta^{2}\right)$, which has a real and positive zero for $\theta^{2}=\chi^{2}$. It can be seen that the parabola $F\left(\theta^{2}\right)$ has two real zeros, one of them positive and the other negative, but only the positive one has physical meaning since $\chi$ is defined as real and then $\chi^{2}$ must be positive. Hence $F\left(\theta^{2}\right)<0$ for $\theta^{2}<\chi^{2}$. In particular, if $[(1+\sigma) / 2]^{2}<\chi^{2}$ (the above instability condition), the 
function $F$ takes a negative value for $\theta^{2}=[(1+\sigma) / 2]^{2}$. Thus, when $\chi^{2}$ is replaced by $[(1+\sigma) / 2]^{2}$ in Eq. (28), we have the instability condition

$$
\begin{aligned}
F\left(\left(\frac{1+\sigma}{2}\right)^{2}\right) & \equiv \frac{\sigma}{4}\left[(1+\sigma)^{2}+\left(\delta-a \sigma r-v k^{2}\right)^{2}-r(1+\sigma)^{2}\right] \\
& <0
\end{aligned}
$$

which can be rearranged to read

$$
G\left(r, k^{2}\right) \equiv(1+\sigma)^{2}(1-r)+\left(\delta-a \sigma r-v k^{2}\right)^{2}<0 .
$$

It is easy to see that the normalized pump $r$ must verify $r$ $>1$ for Eq. (30) to be fulfilled. We can also write the instability condition as a quadratic function of $r$,

$$
\begin{aligned}
G\left(r, k^{2}\right) \equiv & a^{2} \sigma^{2} r^{2}-\left[2 a \sigma\left(\delta-v k^{2}\right)+(1+\sigma)^{2}\right] r+(1+\sigma)^{2} \\
& +\left(\delta-v k^{2}\right)^{2}<0 .
\end{aligned}
$$

This condition is verified for values of $r$ in the range limited by the two real roots. The necessary condition for $G\left(r, k^{2}\right)$ $=0$ to have two different real roots is that the coefficients of the parabola verify

$$
\begin{aligned}
& {\left[2 a \sigma\left(\delta-v k^{2}\right)+(1+\sigma)^{2}\right]^{2}-4 a^{2} \sigma^{2}\left[(1+\sigma)^{2}+\left(\delta-v k^{2}\right)^{2}\right]} \\
& \quad>0
\end{aligned}
$$

which directly leads to

$$
k^{2} \leqslant \frac{1}{v}\left[\delta-a \sigma+\frac{(1+\sigma)^{2}}{4 a \sigma}\right] \equiv k_{\max }^{2} .
$$

Thus, an upper limit to the value of $k$ appears in the instability condition given by Eq. (31). Consequently, only the traveling waves whose values of $k$ are lower than $k_{\max }$ are able to instabilize the nonlasing solution, which means that there is a cutoff in the electric field spectrum. This new result, due to the electronic-vibrational coupling, provides the minimum spatial scale (wavelength $\lambda_{\min } \equiv 2 \pi b / k_{\max }$ ) that can appear in the transverse pattern. This is one of the most interesting consequences of the new terms included in the Maxwell-Bloch equations. It is essential that $k_{\max }^{2}>0$ for $k_{\text {max }}$ to have physical meaning. This last condition applied to Eq. (33) leads to

$$
a<\frac{\delta+\sqrt{\delta^{2}+(1+\sigma)^{2}}}{2 \sigma},
$$

or, what is equivalent [introducing the definition $\delta \equiv \Delta$ $\left.-\left(f_{2}-f_{1}\right) / \gamma_{\perp}\right]$, to

$$
\Delta>\frac{\left(f_{2}-f_{1}\right)}{\gamma_{\perp}}+\frac{\left(f_{2}+f_{1}\right) \sigma}{c \alpha}-\frac{(1+\sigma)^{2}}{4 a \sigma} \equiv \Delta_{\min } .
$$

This new result means that the detuning $\Delta$ must be larger than $\Delta_{\text {min }}$ to obtain laser emission. From Eqs. (33) and (35), for $\lambda_{\min }=2 \pi b / k_{\max }$, we obtain

$$
\lambda_{\min }=\left(\frac{\pi c \lambda}{\gamma_{\perp}\left(\Delta-\Delta_{\min }\right)}\right)^{1 / 2}
$$

When $k<k_{\max }$, i.e., $\lambda>\lambda_{\text {min }}$, the equation $G\left(r, k^{2}\right)=0$ has the two real roots

$$
\begin{aligned}
r_{ \pm}(k)= & \frac{1}{2 a^{2} \sigma^{2}}\left\{2 a \sigma\left(\delta-v k^{2}\right)+(1+\sigma)^{2}\right. \\
& \left. \pm(1+\sigma)^{2} \sqrt{1+\frac{4 a \sigma}{(1+\sigma)^{2}}\left(\delta-a \sigma-v k^{2}\right)}\right\},
\end{aligned}
$$

the nonlasing solution being unstable when the normalized pump verifies $r_{-}(k)<r<r_{+}(k)$. We denote by $r_{b}$ the value where the two branches of $r_{\mathrm{th}}(k)\left[r_{-}(k)\right.$ and $\left.r_{+}(k)\right]$ join,

$$
r_{-}\left( \pm k_{\max }\right)=r_{+}\left( \pm k_{\max }\right)=1+\frac{(1+\sigma)^{2}}{4 a^{2} \sigma^{2}} \equiv r_{b} \text {. }
$$

The laser threshold $r_{\mathrm{th}}(k)$ for any traveling wave (any $k$ ) is obtained by doing $G\left(r_{\text {th }}, k^{2}\right)=0$. The corresponding curve $r_{\text {th }}(k)$ is called the neutral stability curve. In our case $r_{\text {th }}(k)$ consists of the two branches given by Eq. (37), $r_{-}(k)$ and $r_{+}(k)$, in contrast to the case without vibrational coupling, where only the branch $r_{-}(k)$ with $a=0$ appears.

Let first study the branch $r_{-}(k)$. The minimum value of $r_{-}(k)$ occurs for the traveling wave with the lowest threshold (critical wave), that is, for the solution expected at the laser threshold. Its corresponding wave number is termed $k_{-}$. From the derivative $\partial r_{-}(k) / \partial k=0$ we obtain the solutions $k=0$ and $k^{2}=(\delta-a \sigma) / v$. At this point we consider two cases:

(a) $\delta<a \sigma$, i.e., $\Delta<\Delta_{0}$, where

$$
\Delta_{0} \equiv \frac{\left(f_{2}-f_{1}\right)}{\gamma_{\perp}}+\frac{\left(f_{2}+f_{1}\right) \sigma}{c \alpha} \equiv \Delta_{\min }+\frac{(1+\sigma)^{2}}{4 a \sigma}
$$

(the definitions of $\delta$ and $a$ have been used). In this case only the solution $k=0$ has physical meaning, hence $k_{-}=0$ is the solution expected at the laser threshold (see lower part of the curve in Fig. 1). The value of the threshold, termed $r_{-}$, is then

$$
\begin{aligned}
r_{-} \equiv & r_{-}\left(k_{-}=0\right)=\frac{1}{2 a^{2} \sigma^{2}}\left\{(1+\sigma)^{2}+2 a \sigma \delta\right. \\
& \left.-(1+\sigma)^{2} \sqrt{1+\frac{4 a \sigma}{(1+\sigma)^{2}}(\delta-a \sigma)}\right\} .
\end{aligned}
$$

It must be noted that, contrary to the case without electronicvibrational coupling, a negative detuning $\Delta<0$ is not necessary to get the homogeneous wave $\left(k_{-}=0\right)$. In our case this wave can occur even for $\Delta>0$, provided that $\Delta<\Delta_{0}$. Assuming $f_{2}>f_{1}$ (usual case) we obtain $\Delta_{0}>0$, so the homogeneous solution is favored since the detuning $\Delta_{0}$ that sepa- 


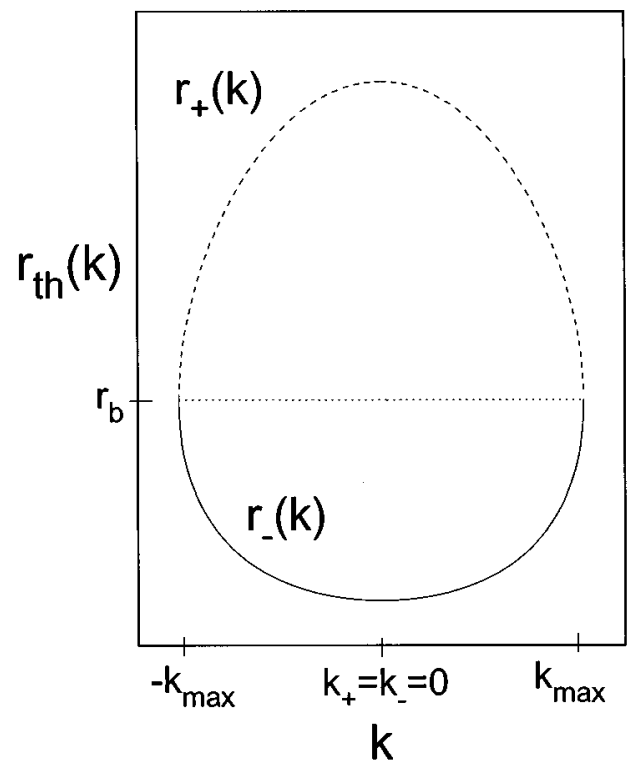

FIG. 1. General shape of branches $r_{-}(k)$ (solid line) and $r_{+}(k)$ (dashed line) of the neutral stability curve vs the wave number $k$, in the case $\Delta_{\min }<\Delta<\Delta_{0}$. The normalized pump $r_{b}$, where the two branches join is shown (dotted line).

rates the two types of solutions shifts to a positive value, increasing the range of $\Delta$ values that lead to the homogeneous solution.

(b) $\delta>a \sigma$, i.e., $\Delta>\Delta_{0}$. In this case, it can be seen that the minimum value of $r_{-}(k)$ is for the other solution $k^{2}=(\delta$ $-a \sigma) / v$, hence $k_{-}=\sqrt{(\delta-a \sigma) / v}$ and traveling waves with $\pm k_{-}$are expected at the laser threshold (see lower part of Fig. 2). The value of the threshold is

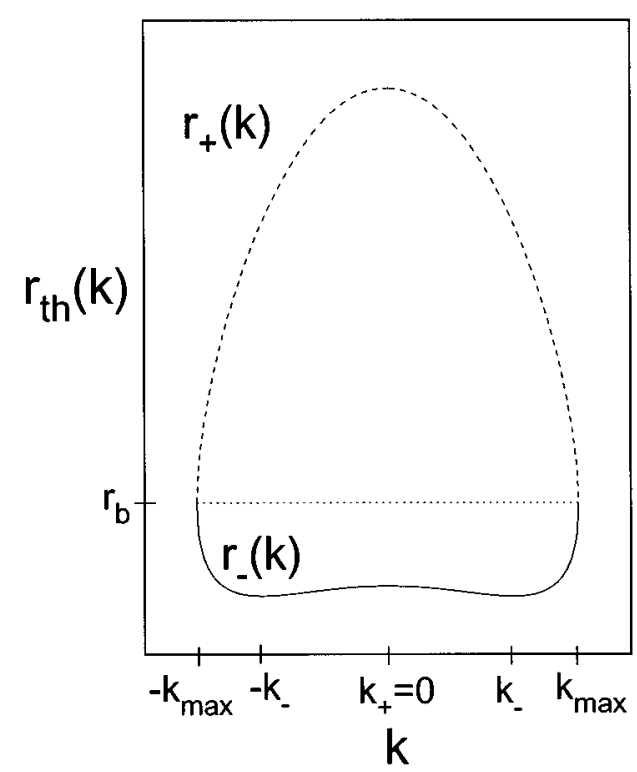

FIG. 2. General shape of branches $r_{-}(k)$ (solid line) and $r_{+}(k)$ (dashed line) of the neutral stability curve vs the wave number $k$, in the case $\Delta>\Delta_{0}>\Delta_{\min }$. The normalized pump $r_{b}$, where the two branches join is shown (dotted line).

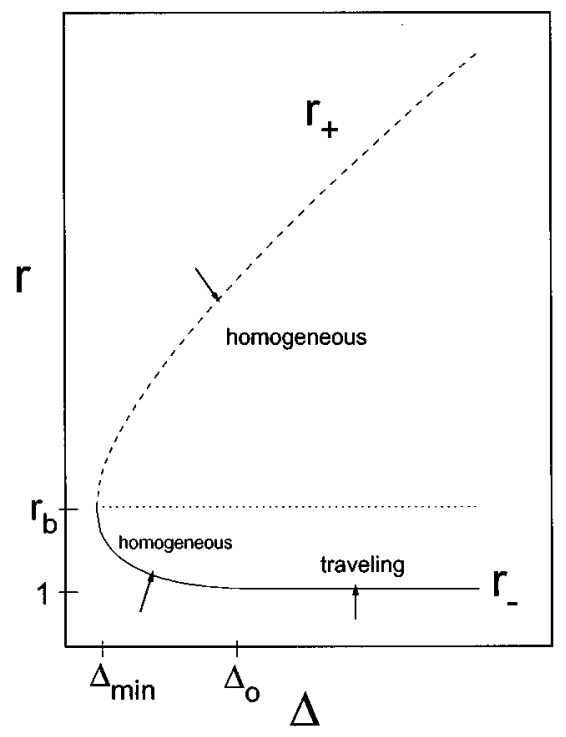

FIG. 3. The first-threshold instability curve in the plane of detuning $\Delta \equiv\left(\omega_{12}-\omega\right) / \gamma_{\perp}$ and normalized pumping $r$. The pump threshold $r_{+}$given by Eq. ( 42) (dashed line) and $r_{-}$given by Eqs. (40) and (41) (solid line). The value of $r_{b}$ is given (dotted line).

$$
r_{-} \equiv r_{-}\left(k_{-}\right)=1
$$

Let now study the branch $r_{+}(k)$ of the neutral stability curve. Diminishing the value of $r$ coming from values $r$ $>r_{+}(k)$, where the nonlasing solution is stable, the first wave that unstabilizes that trivial solution is the wave whose $k_{+}$gives the maximum of the curve $r_{+}(k)$. Since this function has only one maximum at $k=0$, the homogeneous solution, $k_{+}=0$, appears at the laser threshold in this case (see upper part of the curve in Figs. 1 and 2). The value of this threshold is

$$
\begin{aligned}
r_{+} \equiv & r_{+}\left(k_{+}=0\right)=\frac{1}{2 a^{2} \sigma^{2}}\left\{(1+\sigma)^{2}+2 a \sigma \delta\right. \\
& \left.+(1+\sigma)^{2} \sqrt{1+\frac{4 a \sigma}{(1+\sigma)^{2}}(\delta-a \sigma)}\right\} .
\end{aligned}
$$

The general shape of branches $r_{-}(k)$ and $r_{+}(k)$ of the neutral stability curve are shown in Fig. 1 for $\Delta_{\min }<\Delta<\Delta_{0}$, and in Fig. 2 for $\Delta>\Delta_{0}>\Delta_{\text {min }}$.

The first-threshold instability curve in the plane of detuning $\Delta$ and pumping $r$ is shown in Fig. 3. The two branches are $r_{-} \equiv r_{-}\left(k_{-}\right)$, given by Eqs. (40), (41), and $r_{+} \equiv r_{+}\left(k_{+}\right)$, given by Eq. (42). We see from the graphic that when $r_{-}$is reached coming from lower values than $r_{-}$, the solution at threshold is homogeneous or traveling depending on the $\Delta$ value, while the solution is always the homogeneous one when $r$ diminishes coming from higher values than $r_{+}$to reach $r_{+}$.

Let us now consider a numerical example where the molecular data are representative of substituted aromatic molecules that are normally used as active centers in lasers [25]. Different cases with equal or different coupling values $f_{1}$ 


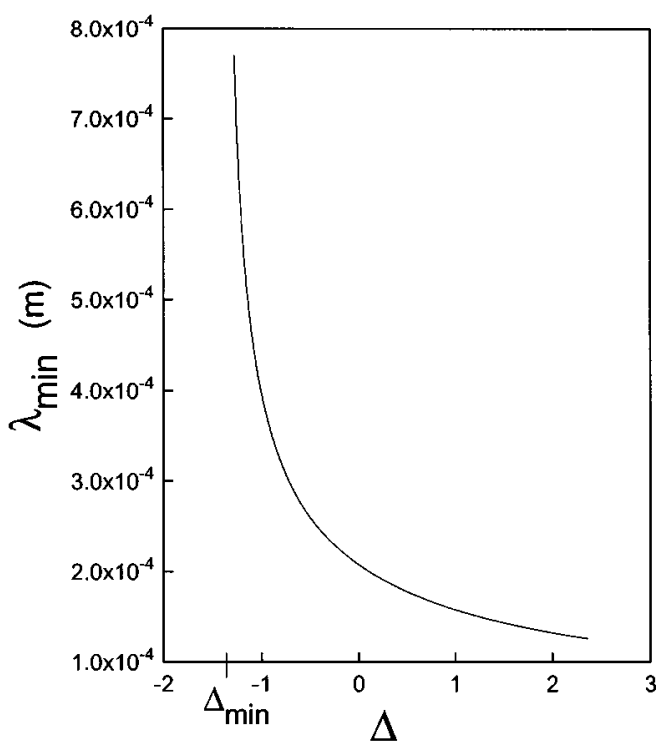

FIG. 4. The minimum wavelength $\lambda_{\min }$ vs dimensionless detuning $\Delta$ for the parameters $\omega_{12}=3.0 \times 10^{15} \mathrm{~s}^{-1}, \mu_{12}=0.1 \mathrm{D}$ $=0.33695 \times 10^{-30} \mathrm{C} \mathrm{m}, \gamma_{\|}=10^{9} \mathrm{~s}^{-1}, \gamma_{\perp}=10^{10} \mathrm{~s}^{-1}, N=5.0 \times 10^{22}$ $\mathrm{m}^{-3}, f_{2}=2.0 \times 10^{12} \mathrm{~s}^{-1}, f_{1}=1.9999 \times 10^{12} \mathrm{~s}^{-1}, L=0.02 \mathrm{~m}, b$ $=0.01 \mathrm{~m}, R_{1}=0.9, R_{2}=1$, and $\sigma_{c} / \epsilon_{0} \simeq-c \ln \sqrt{R_{1} R_{2}} / L$.

and $f_{2}$ can be found among the wide variety of molecules. In general, $f_{2} \geqslant f_{1}$ can be assumed. Let us take $\omega_{12}=3.0$ $\times 10^{15} \mathrm{~s}^{-1}, \quad \mu_{12}=0.1 \mathrm{D}=0.33695 \times 10^{-30} \mathrm{C} \mathrm{m}, \gamma_{\|}=10^{9}$ $\mathrm{s}^{-1}, \gamma_{\perp}=10^{10} \mathrm{~s}^{-1}, N=5.0 \times 10^{22} \mathrm{~m}^{-3}, f_{2}=2.0 \times 10^{12} \mathrm{~s}^{-1}$, $f_{1}=1.9999 \times 10^{12} \mathrm{~s}^{-1}$, and the following resonator parameters $L=0.02 \mathrm{~m}, b=0.01 \mathrm{~m}, R_{1}=0.9, R_{2}=1$, and $\sigma_{c} / \epsilon_{0}$ $\simeq-c \ln \sqrt{R_{1} R_{2}} / L$. With these values we obtain $\Delta_{\min }=$ -1.38 and $\Delta_{0}=0.18$. We must take into account that the detuning $\left(\omega_{12}-\omega\right)$ must be less than half of the axial-mode spacing of the resonator, $\pi c /(2 L)$, i.e., $|\Delta| \leqslant \pi c /\left(2 L \gamma_{\perp}\right)$ $=2.356$. In spite of this restriction, it is possible in this case to get the two different spatial solutions at threshold, homogeneous wave for $\Delta_{\min }=-1.38<\Delta<\Delta_{0}=0.18$, and traveling wave for $\Delta>\Delta_{0}$.

Using the above data we represent in Fig. 4 the minimum wavelength $\lambda_{\min }$ that can appear in the system, versus detuning [Eq. (36)], its value being approximately $\lambda_{\min } \simeq 200 \mu \mathrm{m}$ for $\Delta$ values not very close to $\Delta_{\min }$.

We must note that the detuning $\Delta_{0}$, which separates the two transverse spatial patterns, depends on the physical parameters of the medium and cavity. In Fig. 5 we represent $\Delta_{0}$ versus $N$, for the molecular and laser parameters given above. It can be seen from the graphic how we can select different types of patterns at threshold by changing the number of molecules per unit volume, the detuning remaining fixed.

Figure 6 represents $\Delta_{0}$ versus the difference $f_{2}-f_{1}$ for fixed $f_{1}=1.9999 \times 10^{12} \mathrm{~s}^{-1}$. The restriction of the detuning imposed by the axial-mode spacing is indicated. It can be seen that when $\Delta_{0}$ is higher than this value, only the homogeneous solution is possible at the first threshold.

\section{SOLUTION ABOVE THRESHOLD. NATURE OF THE BIFURCATION}

The system admits, above threshold, traveling wave solutions of the form

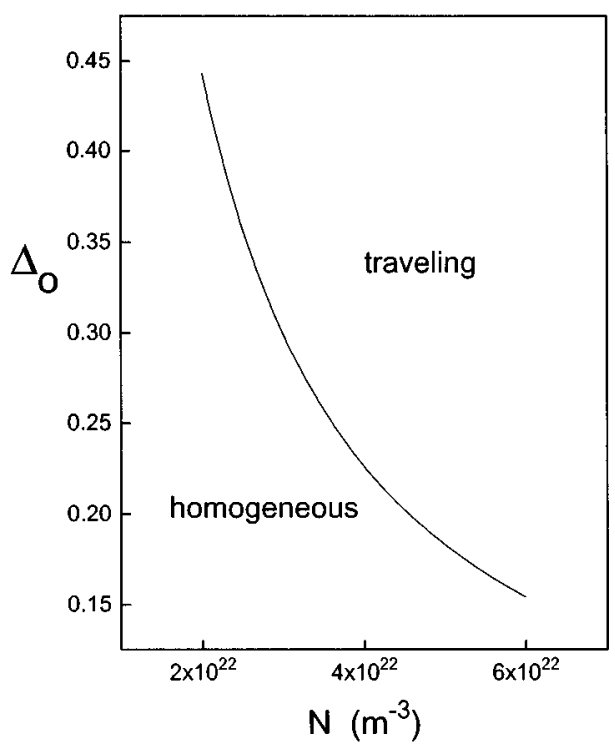

FIG. 5. The critical dimensionless detuning $\Delta_{0}$ vs $N$ for the same parameters as in Fig. 4.

$$
E_{s}=\bar{e} e^{i(\vec{k} \cdot \vec{\rho}+\bar{\omega} \tau)}, \quad P_{s}=\bar{p} e^{i(\vec{k} \cdot \vec{\rho}+\bar{\omega} \tau)}, \quad D_{s}=\bar{d},
$$

where $\bar{e}$ and $\bar{d}$ are real numbers whereas $\bar{p}$ is a complex quantity. Introducing the expressions (43) in Eqs. (19)-(21) we found that the amplitude $\bar{e}$ of the electric field is solution of the equation

$$
\begin{aligned}
& a^{2} \sigma^{2} e^{4}+\left[(1+\sigma)^{2}+2\left(\delta-a \sigma r-v k^{2}\right) a \sigma\right] \bar{e}^{2} \\
& +(1+\sigma)^{2}(1-r)+\left(\delta-a \sigma r-v k^{2}\right)^{2}=0,
\end{aligned}
$$

$\bar{\omega}, \bar{d}$ and $\bar{p}$ verifying

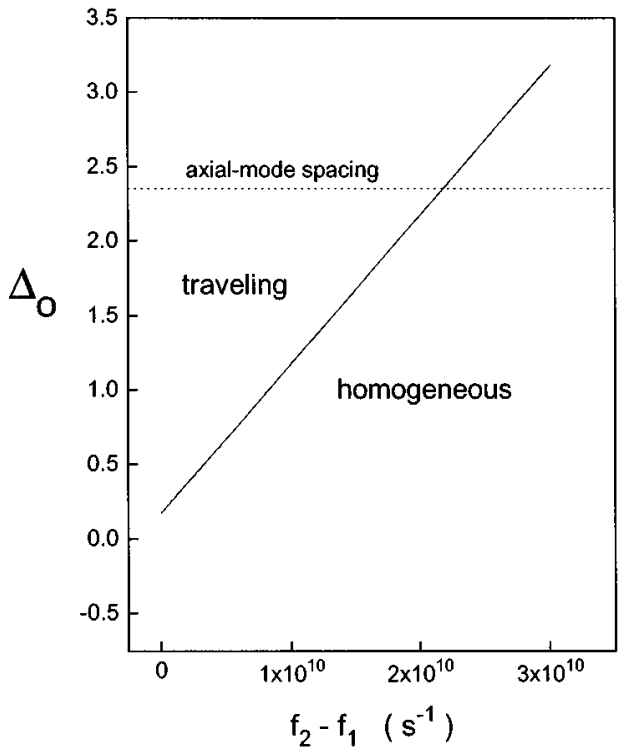

FIG. 6. The critical detuning $\Delta_{0}$ vs the difference $f_{2}-f_{1}$ for the same parameters as in Fig. 4 and $f_{1}$ remaining fixed at 1.9999 $\times 10^{12} \mathrm{~s}^{-1}$. The dimensionless detuning (2.356) imposed by the axial-mode spacing is shown (dotted line). 


$$
\begin{gathered}
\bar{\omega}=\frac{a \sigma^{2}\left(r-\bar{e}^{2}\right)-\sigma \delta-v k^{2}}{1+\sigma}, \quad \bar{d}=r-\bar{e}^{2}, \\
\bar{p}=\left(1+i \frac{\bar{\omega}+v k^{2}}{\sigma}\right) \bar{e} .
\end{gathered}
$$

Equation (44), of second degree in $\bar{e}^{2}$, admits two solutions for the variable $\bar{e}^{2}$,

$$
\overline{e_{ \pm}^{2}}=r-r_{ \pm}(k)
$$

It can be seen from Eq. (46) that in the region where the nonlasing solution is unstable, i.e., for $r_{-}(k)<r<r_{+}(k)$, only the solution $\vec{e}_{-}^{2}$ has physical meaning. Following [18], the bifurcation is subcritical if one of the solutions given by Eq. (46) exists in the nonlasing region, where the nonlasing state is stable $\left[r<r_{-}(k)\right.$ and $\left.r>r_{+}(k)\right]$. Both solutions $\left(\bar{e}_{ \pm}^{2}\right)$ exist above $r_{+}(k)$, so the bifurcation through this branch is subcritical. However, since there is not any solution below $r_{-}(k)$, the bifurcation through this branch is supercritical, as in the case without vibrational coupling [18].

\section{LINEAR STABILITY ANALYSIS OF THE LASING SOLUTION}

We study in this section the stability of the critical traveling wave $k=\sqrt{(\delta-a \sigma) / v}$ and the homogeneous wave $k$ $=0$, these solutions being the two different possible patterns at the first laser threshold.

Using standard linear-stability methods we introduce small perturbations in the solution given by Eq. (43),

$$
\begin{gathered}
E_{s}=(\bar{e}+\delta e) e^{i(\vec{k} \cdot \vec{\rho}+\bar{\omega} \tau)}, \quad P_{s}=(\bar{p}+\delta p) e^{i(\vec{k} \cdot \vec{\rho}+\bar{\omega} \tau)}, \\
D_{s}=\bar{d}+\delta d,
\end{gathered}
$$

where $\delta e, \delta p$, and $\delta d$ are the perturbations, and $k$ is the wave number of the traveling or the homogeneous wave. To obtain the linearized system we assume plane waves for $\delta e$ and $\delta p$, of dimensionless wave vector $\vec{q}$, so that $\vec{\nabla}(\delta e)$ $=i \vec{q}(\delta e)$, and analogously for $\delta p$. The linearized system obtained for the perturbations is

$$
\begin{aligned}
\frac{\partial(\delta e)}{\partial \tau}=- & {\left[\sigma+i \bar{\omega}+i v(\vec{k}+\vec{q})^{2}\right](\delta e)+\sigma(\delta p) } \\
\frac{\partial(\delta p)}{\partial \tau}= & \bar{d}(\delta e)-[1+i(\delta+\bar{\omega}-a \sigma \bar{d})](\delta p) \\
& +(\bar{e}+i a \sigma \bar{p})(\delta d) \\
\frac{\partial(\delta d)}{\partial \tau}=- & \gamma\left[(\delta d)+\frac{1}{2}\left[\bar{p}^{*}(\delta e)+\bar{p}\left(\delta e^{*}\right)+\bar{e}(\delta p)\right.\right. \\
& \left.\left.+\bar{e}\left(\delta p^{*}\right)\right]\right]
\end{aligned}
$$

Setting $\delta e=e_{1}+i e_{2}$ and $\delta p=p_{1}+i p_{2}$, we finally obtain the five-dimensional system for the real and imaginary parts of $\delta e, \delta p$, and for $\delta d$,

$$
\begin{gathered}
\partial_{\tau} e_{1}=-\sigma e_{1}+\sigma p_{1}+\left[\omega+v(\vec{k}+\vec{q})^{2}\right] e_{2} \\
\partial_{\tau} p_{1}=\left(r-\bar{e}^{2}\right) e_{1}-p_{1}+\left[\delta+\bar{\omega}-a \sigma\left(r-\bar{e}^{2}\right)\right] p_{2} \\
+\bar{e}\left[1-a\left(\bar{\omega}+v k^{2}\right)\right](\delta d) \\
\partial_{\tau} e_{2}=-\left[\bar{\omega}+v(\vec{k}+\vec{q})^{2}\right] e_{1}-\sigma e_{2}+\sigma p_{2} \\
\partial_{\tau} p_{2}=-\left[\delta+\bar{\omega}-a \sigma\left(r-\bar{e}^{2}\right)\right] p_{1}+\left(r-\bar{e}^{2}\right) e_{2}-p_{2} \\
+a \sigma \bar{e}(\delta d) \\
\partial_{\tau}(\delta d)=-\gamma \bar{e} e_{1}-\gamma \bar{e} p_{1}-\frac{\gamma \bar{e}}{\sigma}\left(\bar{\omega}+v k^{2}\right) e_{2}-\gamma(\delta d)
\end{gathered}
$$

The laser solution is stable if, for all values of $q$, the matrix of the coefficients of the above system has all its eigenvalues with negative real part. If any eigenvalue has positive real part, the solution is unstable. The problem of finding the eigenvalues of the $5 \times 5$ matrix has been approached numerically.

We will consider the two types of instability most common in the Maxwell-Bloch laser equations: The Eckhaus instability, occurring along the direction of the traveling wave ( $\vec{q}$ parallel or antiparallel to $\vec{k}$ ), and the zigzag instability, occurring at right angles of the traveling wave $(\vec{q}$ perpendicular to $\vec{k}$ ).

We use in the following numerical example the same data as in Sec. III and $r$ values up to $r=50$. Cases with and without electronic-vibrational coupling will be compared. First we consider the case without such a coupling, i.e., $f_{1}$ $=f_{2}=0$ and then $a=0, \delta=\Delta$. We found, in agreement with other authors $[17,18]$, that for positive detuning, the critical traveling wave is stable and the homogeneous wave is unstable, while for negative detuning, the homogeneous wave is stable (see Fig. 7).

In the case where the electronic-vibrational coupling is taken into account, there are two cases, (a) and (b) (as we stated in Sec. III).

(a) $\Delta_{\min }<\Delta<\Delta_{0}=0.18$ : In this case only the homogeneous solution is possible, being stable in a wide range of $r$ and $\Delta$ values [see Fig. 8(a)]. Note that in the case of positive detuning, the behavior is different from the case without vibrational coupling.

To see how the homogeneous solution becomes unstable we show in Fig. 9(a) the growth rate curve for the perturbation versus the wave number $q$. The solution becomes unstable following both Eckhaus and zigzag instabilities for $q$ $\simeq 120$ (both curves have the same shape). This instability behavior is similar to that of the case without vibrational coupling.

(b) $\Delta>\Delta_{0}=0.18$ : The critical traveling wave is the one selected at threshold. However, this solution becomes unstable above values of $r$ very close to the threshold [see Fig. $8(\mathrm{~b})$ ], while the homogeneous wave is stable and survives at higher $r$ values for $\Delta$ not very far from $\Delta_{0}$ [see Figs. 8(a) and $8(b)]$. So then, only for very low $r$ values, the cases with and without vibrational coupling present a rather similar behavior (the critical traveling wave is stable). This traveling wave disappears following an Eckhaus instability [see Fig. 9(b)]. This instability appears around two different $q$ values; 


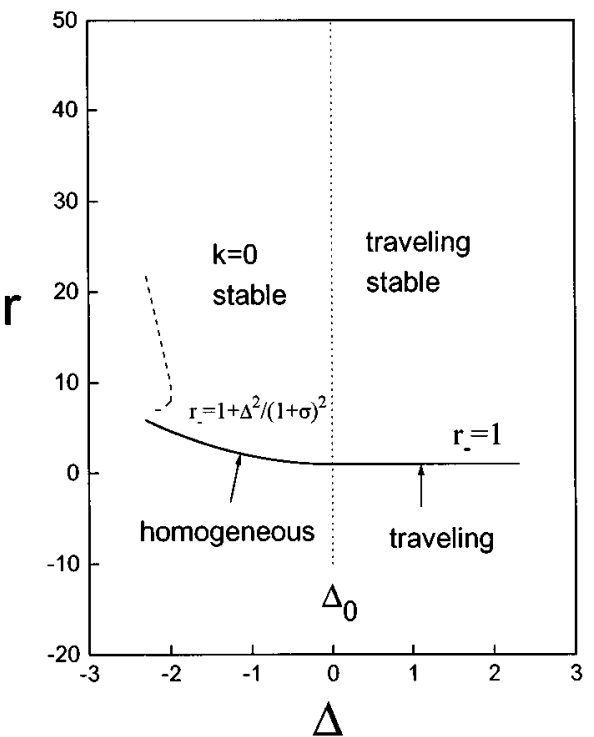

FIG. 7. The first-threshold instability curve (solid line) and the second-threshold instability curve (dashed line) of the homogeneous and the critical traveling wave, in the plane of detuning $\Delta$ and normalized pumping $r$, without vibrational coupling. The parameters are the same as in Fig. 4 but now $f_{1}=f_{2}=0$. The detuning $\Delta_{0}=0$ (dotted line) that separates the two transversal spatial patterns is shown.

at long wavelength $(q \simeq 0)$ and at shorter wavelength ( $q$ $\simeq 130$ ). The homogeneous wave becomes unstable at high $\Delta$ values [see Fig. 8(a)], following Eckhaus and zigzag instabilities [see Fig. 9(c)]. The instabilities appear at short wavelength, $q \simeq 350$, both growth curves having the same shape.

\section{CONCLUSIONS}

Electronic-vibrational coupling in the two-level active centers of a single-mode laser has been analyzed assuming a linear coupling between the electronic states (the ground and the excited states) and the normal vibrational modes.

This coupling leads to two new terms in the semiclassical Maxwell-Bloch equations. One of the terms leads to a slight shift in energy in the two electronic levels. The other term, nonlinear, can be interpreted well as a detuning depending on the population inversion, as well as an additional field proportional to the polarization.

The neutral stability curve depends now on the coupling parameters. In this situation, it is found that the instability condition of the nonlasing solution provides the minimum wavelength or spatial scale that can appear in the transverse pattern. Such a minimum wavelength depends on the coupling parameters, detuning and laser parameters. For typical data of the laser with substituted aromatic molecules as active centers, it is found that $\lambda_{\min } \simeq 200 \mu \mathrm{m}$.

The neutral stability curve $r_{\mathrm{th}}(k)$ has two branches, $r_{-}(k)$ and $r_{+}(k)$, in contrast to the case without vibrational coupling, where only the branch $r_{-}(k)$ appears. In this branch $r_{-}(k)$, the selected spatial state is the homogeneous wave $(k=0)$ when the detuning $\Delta \equiv\left(\omega_{12}-\omega\right) / \gamma_{\perp}$ verifies $\Delta_{\text {min }}$

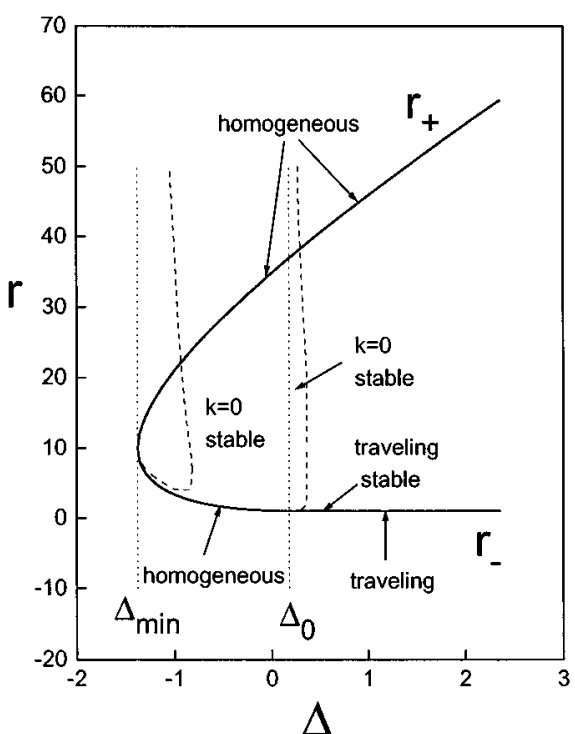

(a)

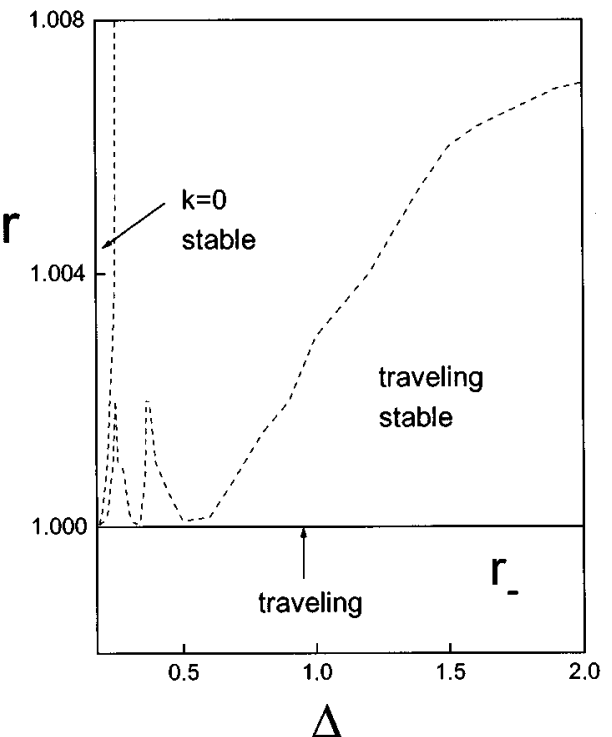

(b)

FIG. 8. (a) The first-threshold instability curve (solid line) and the second-threshold instability curve (dashed line) of the homogeneous and the critical traveling wave, in the plane of detuning $\Delta$ and normalized pumping $r$, for the same parameters as in Fig. 4. The detuning $\Delta_{0}=0.18$ (dotted line) that separates the two transversal spatial patterns, and the lower bound $\Delta_{\min }=-1.38$ (dotted line) that appears in the instability condition are shown. (b) Detail of Fig. 8(a) in the region $\Delta>\Delta_{0}$ and $r$ values close to the first threshold.

$<\Delta<\Delta_{0}$, and traveling wave when $\Delta>\Delta_{0}$, where $\Delta_{\min }$ and $\Delta_{0}$ are values depending on the coupling parameters and laser parameters. A lower bound for detunings, $\Delta_{\min }$, appears in the instability condition. This bound limits the allowed detunings to obtain laser emission. The corresponding pump threshold values are given. Contrary to the case without vibrational coupling [16-19], here a negative detuning is not necessary to get the homogeneous wave at the first threshold since $\Delta$ can be positive provided that $\Delta<\Delta_{0}$. So then, the homogeneous wave becomes favored, being the only solution allowed in some cases. A similar theoretical result has been found recently when certain losses are introduced in the Maxwell-Bloch equations [20]. 


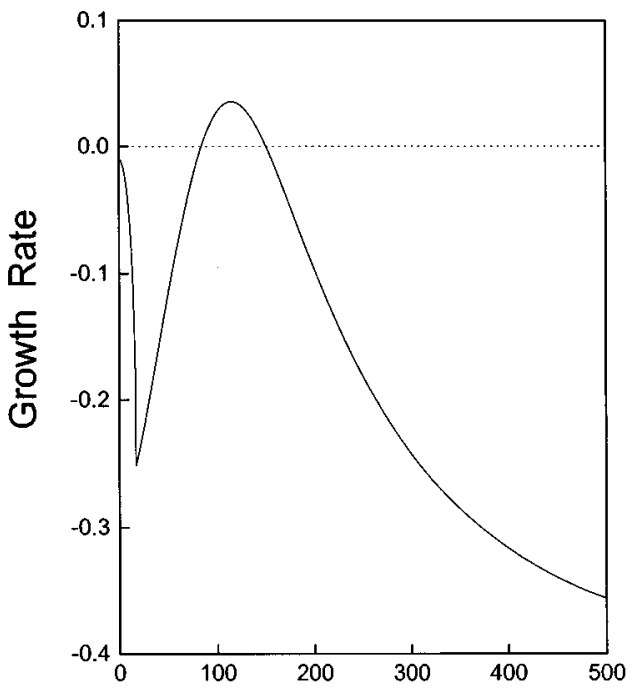

(a) q

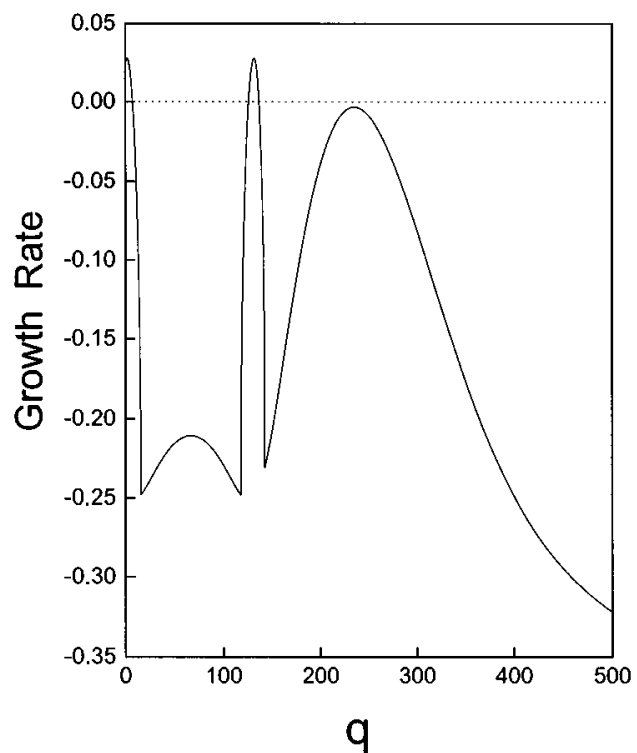

(b)

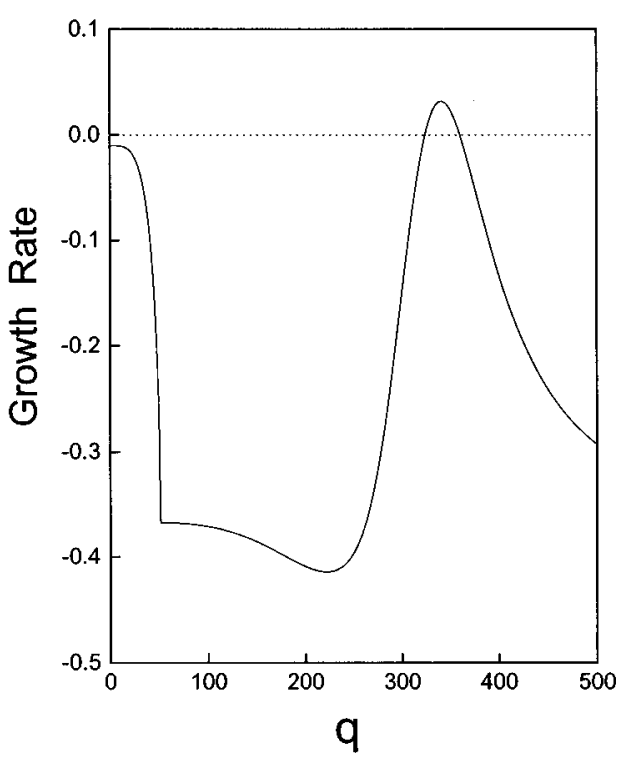

FIG. 9. The largest real part of any eigenvalue as a function of the dimensionless wave number $q$, for the same parameters as in Fig. 4 . (a) Eckhaus ( $\vec{q}$ parallel or antiparallel to $\vec{k})$ and zigzag $(\vec{q}$ perpendicular to $\vec{k})$ instability for the homogeneous wave $k=0 ; r=10$ and $\Delta$ $=-1$. (b) Eckhaus instability ( $\vec{q}$ parallel or antiparallel to $\vec{k}$ ) for the critical traveling wave with $k=66.69 ; r=2$ and $\Delta=0.25$. (c) Eckhaus $(\vec{q}$ parallel or antiparallel to $\vec{k})$ and zigzag $(\vec{q}$ perpendicular to $\vec{k})$ instability for the homogeneous wave $k=0 ; r=25$ and $\Delta=0.36$.

It is found that the new branch, $r_{+}(k)$, of the neutral stability curve always leads to the selection of the homogeneous wave at threshold. Its pump value is given.

We study the behavior of the critical detuning $\Delta_{0}$ [which separates the two types of patterns at threshold in branch $\left.r_{-}(k)\right]$ with the concentration of the active centers and with the vibrational coupling parameters.

The bifurcation at threshold was found to be supercritical in branch $r_{-}(k)$ (as in the case without vibrational coupling) and subcritical in the new branch $r_{+}(k)$.

The stability of the patterns selected at the first threshold, the homogeneous wave $(k=0)$ and the critical traveling wave $k=\sqrt{(\delta-a \sigma) / v}$, has been analyzed.

For $\Delta<\Delta_{0}$, the homogeneous wave is stable in a wide range of $r$ and $\Delta$ values.

For $\Delta>\Delta_{0}$, the critical traveling wave is stable only for $r$ values very close to the threshold. For higher $r$ values, the homogeneous wave becomes stable in a range of $\Delta$ values not very far from $\Delta_{0}$. So, the homogeneous wave is the only one that can survive for high $r$ values.

It can be said that the electronic-vibrational coupling favors the homogeneous pattern to the detriment of the traveling one, due to the shift of the detuning $\Delta_{0}$ to a positive value, and because the homogeneous solution is the only one that can be stable for high pumping values.

\section{ACKNOWLEDGMENTS}

We are very grateful to J. M. Guerra for his helpful advice. This work was supported by the DGICYT Project No. PB95-0389 (Spain). 
[1] C. Cojan, G. Agrawal, and C. Flytzanis, Phys. Rev. B 15, 909 (1977).

[2] B. P. Antoniuk, Solid State Commun. 33, 873 (1980).

[3] G. J. Blanchard, Phys. Rev. Lett. 63, 887 (1989).

[4] N. Ba and N. T. Dan, Phys. Lett. A 136, 71 (1989).

[5] X. S. Li, D. L. Lin, and T. George, Phys. Rev. B 41, 3280 (1990)

[6] I. Gonzalo, M. A. Antón, and J. L. Escudero, IEEE J. Quantum Electron. 28, 765 (1992).

[7] M. A. Antón and I. Gonzalo, IEEE J. Quantum Electron. 31, 1088 (1995).

[8] O. G. Calderón and I. Gonzalo, Opt. Commun. 125, 369 (1996).

[9] M. Brambilla, M. Cateneo, L. A. Lugiato, R. Pirovano, F. Prati, A. J. Kent, G. L. Oppo, A. B. Coates, C. O. Weiss, C. Green, E. J. D'Angelo, and J. R. Tredicce, Phys. Rev. A 49, 1427 (1994).

[10] A. B. Coates, C. O. Weiss, C. Green, E. J. D’Angelo, J. R. Tredicce, M. Brambilla, M. Cataneo, L. A. Lugiato, R. Pirovano, F. Prati, A. J. Kent, and G. L. Oppo, Phys. Rev. A 49, 1452 (1994).

[11] F. Prati, M. Brambilla, and L. A. Lugiato, Riv. Nuovo Cimento 17, 1 (1994).

[12] O. G. Calderón, V. M. Pérez-García, I. Martin, and J. M. Guerra, Phys. Rev. A 53, 3490 (1996).
[13] V. M. Pérez-García and J. M. Guerra, Phys. Rev. A 50, 1646 (1994).

[14] V. M. Pérez-García, I. Pastor, and J. M. Guerra, Phys. Rev. A 52, 2392 (1995).

[15] A. C. Newell and J. V. Moloney, Nonlinear Optics (Addison Wesley, Redwood City, CA, 1992).

[16] P. Coullet, L. Gil, and F. Rocca, Opt. Commun. 73, 403 (1989).

[17] P. K. Jakobsen, J. V. Moloney, A. C. Newell, and R. Indik, Phys. Rev. A 45, 8129 (1992).

[18] P. K. Jakobsen, J. Lega, Q. Feng, M. Staley, J. V. Moloney, and A. C. Newell, Phys. Rev. A 49, 4189 (1994).

[19] J. Lega, P. K. Jakobsen, J. V. Moloney, and A. C. Newell, Phys. Rev. A 49, 4201 (1994).

[20] O. G. Calderón, V. M. Pérez-García, J. Lega, and J. M. Guerra, Opt. Commun. 1443, 315 (1997).

[21] B. P. Antoniuk, Zh. Éksp. Teor. Fiz. 80, 2221 (1981) [Sov. Phys. JETP 53, 1159 (1981)].

[22] D. Pines, Elementary Excitations in Solids (Benjamin, New York, 1964).

[23] D. B. Fitchen, in Physics of Color Centers, edited by W. B. Fowler (Academic, New York, 1968).

[24] H. Haken, Light Vol. 2: Laser Light Dynamics (North-Holland, Amsterdam, 1985).

[25] N. C. Kothari and Takayoshi Kobayashi, IEEE J. Quantum Electron. 20, 418 (1984). 